\title{
Design and fabrication of a holey fiber microfluidic device with transverse micro-channel
}

\author{
Dora Juan Juan Hu, ${ }^{* 1}$ Ying Cui, ${ }^{2,3,4}$, Jun Long Lim,${ }^{1,2}$ Perry Ping Shum, ${ }^{2}$ Georges Humbert ${ }^{3}$, Jean-Louis Auguste ${ }^{3}$ \\ and Yixin Wang ${ }^{1}$ \\ ${ }^{l}$ Institute for Infocomm Research, Agency for Science, Technology and Research (A*STAR), Singapore \\ ${ }^{2}$ School of Electrical and Electronic Engineering, Nanyang Technological University, Singapore \\ ${ }^{3}$ XLIM research institute UMR CNRS University of Limoges, France \\ ${ }^{4}$ CINTRA CNRS/NTU/THALES, UMI 3288, Research Techno Plaza, 50 Nanyang Drive, Border X Block, \\ Level 6, Singapore 637553
}

Received June 15, 2014; accepted June 25, 2014; published June 30, 2014

\begin{abstract}
In this report, we present the design of a holey fiber structure with six air holes in the cladding and a transverse microfluidic channel intersecting the silica core for the purpose of liquid detection. The simulation results suggest an improved refractive index sensitivity of the proposed structure compared to that of a single mode fiber with a similar transverse channel. The proposed holey fiber structures are fabricated and characterized.
\end{abstract}

The holey structures within photonic crystal fibers (PCFs) have been extensively exploited in literature to address versatile challenges and opportunities in telecommunications such as dispersion engineering [1-2], nonlinear optics [3-4], sensing applications of refractive index [5-6], biological events [7-8], and temperature [911] etc. The presence of air holes facilitates the development of a robust fiber-based optofluidic platform using PCFs. There are two common forms of optofluidic sensing based on PCFs. The first one is utilizing the air channels to flow the fluidic analyte and the second one is only taking advantage of controllable light-matter interaction in a PCF structure without filling analyte into air holes. One obvious advantage of air channel-based optofluidic PCF devices is that the required liquid volume inside the air channels is much lower than PCF sensors developed for external sensing, usually in the order of femtoliter to subnanoliter depending on the infiltrated air channel diameter and the infiltration length [12]. The infiltration to the air channel can be unselective so that all air channels are filled by liquid [12] or selective with only specific air channel(s) filled by liquid [9-11]. On the other hand, flowing analyte into air channels imposes a practical challenge of purging and reusing the device. Therefore, in practice, the air channel-based microfluidic PCF device is made for one-time use only. PCF devices for external sensing can be reused easily by purging the surface by a suitable liquid [8]. In this paper, we propose a holey structure which combines the advantage of

*E-mail: jjhu@i2r.a-star.edu.sg external sensing and minimizes liquid sample volume. The holey fiber has six air holes in the cladding which are not filled by liquid analyte, thus eliminating the complexity in washing the air channels and enabling its integration into an all fiber system by simply splicing both fiber ends to standard fibers making this device compact and robust. A transverse channel is inserted across the fiber core, allowing maximum interaction between light mode and liquid analyte in the channel while maintaining sufficient optical power for detection. The cross-section, top and side schematic of the proposed device are depicted in Fig. 1. The microchannel can be fabricated by drilling it, using a specific setup based on femtosecond laser technology [13], which is an efficient approach to precisely fabricate engineered structures on silica fibers.

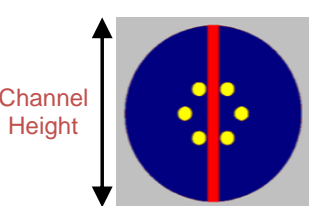

(a)

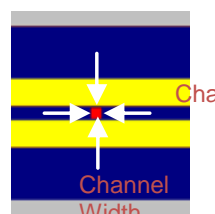

(b)

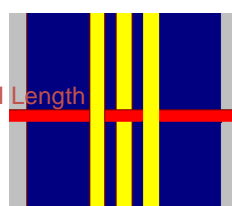

(c)
Fig. 1. (a) Cross-section, (b) top and (c) side view of the proposed microfluidic PCF design. Blue indicates silica, yellow indicates holey region, and red indicates the microfluidic channel.

For allowing the insertion of a transverse channel across the fiber core, the topology of the holey fiber is limited to a silica core surrounded by six air channels running axially along the fiber axis. The six air holes are arranged in a hexagon pattern, forming an effective cladding with a lower refractive index than that of the silica core. The microfluidic channel is designed to cross the fiber core without touching the neighbouring voids. The channel width is $8 \mu \mathrm{m}$, channel height (transverse of the fiber) is $125 \mu \mathrm{m}$, the channel length (along the fiber axis) is $8 \mu \mathrm{m}$. The channel size leads to minimal silica strut thickness of $10 \mu \mathrm{m}$. 
In order to meet these requirements, the attenuation coefficient of the fundamental mode has been simulated versus the hole diameter and for a different pitch $(\Lambda)$, i.e. air hole spacing. The results are shown in Fig. 2. As expected, six air holes lead to weak light confinement within the core and the loss is reduced with increasing hole diameters (i.e. reducing strut thickness).

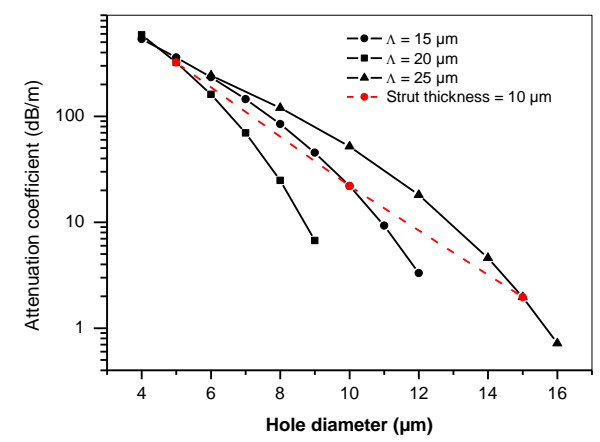

Fig. 2. Evolution versus the air hole diameter of the attenuation coefficient of the fundamental mode of a holey fiber composed of six air holes.

Even if the attenuation coefficient could be further reduced by increasing the pitch, a pitch of $20 \mu \mathrm{m}$ and hole diameter of $10 \mu \mathrm{m}$ are chosen as the best compromise. Larger core size increases further fiber bending losses and mode field mismatch with the fundamental mode of standard fiber leading to larger splicing losses. A large attenuation coefficient of $22 \mathrm{~dB} / \mathrm{m}$ (at $1550 \mathrm{~nm}$ ) is not a strong limitation since only a short fiber length (few centimetres) is used in the device.

The holey fiber is fabricated in XLIM research institute, using the "stack and draw" technique. The photograph of the stack and fiber cross section is shown in Fig. 3.
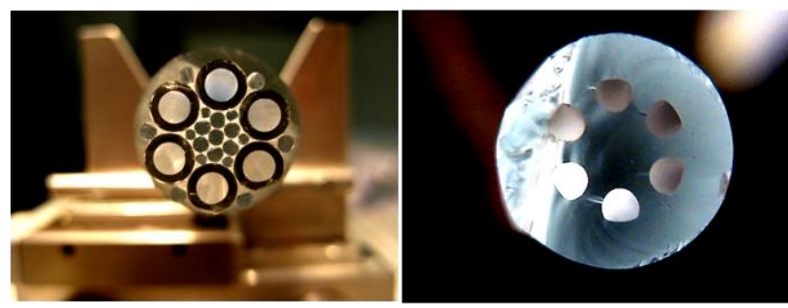

Fig. 3. Stack and cross section view of the holey fiber.

In total, six holey structures with a different dimension are fabricated, named as $\mathrm{N} i, i=1$ to $6 . \mathrm{N} 1$ resembles the optimized design while N2 to N6 show more discrepancy. Therefore they can be used to provide comparative investigation with the optimized structure as well as useful feedback of adjustments of fabrication parameters. Samples of fabricated fibers, are spliced to SMF in a transmission configuration with one side connected to a a broadband light source and the other side connected to an optical spectrum analyzer. The measured spectra are plotted in Fig. 4. The splices of all fiber samples to SMF are repeatable. We observe different insertion losses. The best insertion losses are $4 \mathrm{~dB}$ at $1565 \mathrm{~nm}$ and $1579 \mathrm{~nm}$ for N2 and N6, respectively. N1 exhibits the highest insertion loss of the six samples but it achieves the best extinction ratio. The summary of the extinction ratio of the transmission spectra for all the fabricated fibers is presented in the table. The interference suggests that multiple modes are propagating in the PCF samples. These experimental results demonstrate that the designed holey fiber is a suitable for developing an all-fiber integrated microfluidic device with a transverse microchannel.

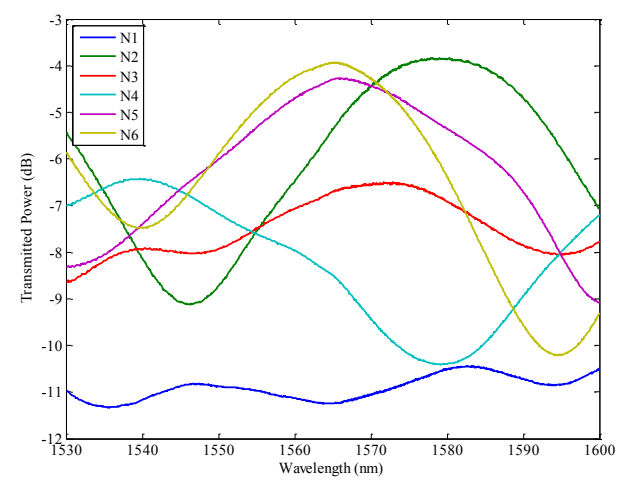

Fig. 4. Transmission spectrum for N1 to N6 PCF. The PCF length used is $\sim 20 \mathrm{~mm}$.

Table. Summary of the extinction ratio of the fiber samples

\begin{tabular}{|l|l|l|l|l|l|l|}
\hline & N1 & N2 & N3 & N4 & N5 & N6 \\
\hline Fiber length (mm) & 21.0 & 19.5 & 19.5 & 18.5 & 19.5 & 20.0 \\
\hline Extinction (dB) & 0.41 & 5.30 & 1.53 & 4.00 & 4.80 & 3.57 \\
\hline
\end{tabular}

The evolution of the intensity distribution of the fundamental mode profile and its propagation in the microfluidic holey fiber are investigated using RSOFT BeamPROP software and shown in Fig. 5 and Fig. 6. In the simulation, the parameters used are as follows: silica refractive index is 1.44, material dispersion is not included in the calculation for simplicity, the outer diameter of the fiber is $125 \mu \mathrm{m}$, air hole diameter is 10 $\mu \mathrm{m}$, air hole spacing is $20 \mu \mathrm{m}$, the wavelength is $1.55 \mu \mathrm{m}$. As expected, the intensity distribution of the fundamental mode is shaped by the hexagon pattern of the air holes as shown in Fig. 5(a). Figure 5(b) presents the mode evolution of the holey structure with a microchannel along the propagation across the microchannel. The propagating core mode is guided in the holey fiber until it encounters the microchannel which causes disturbance of the mode confinement within the core region. Light is scattered into the cladding and reflected at the cladding/surrounding interface back to the core with losses. The microchannel acts as a Fabry-Perot cavity and the transmitted light experiences multiple reflections at core/channel interfaces on both sides. After exiting the channel, partial light is travelling in the cladding which experiences refractions and reflections at the 
cladding/surrounding interface. If the microchannel is filled with a fluid for detection, the interaction of light and fluid occurs at both the core/channel interface and outer cladding/surrounding interface. By monitoring the transmitted power, the refractive index of the fluids in the channel and surrounding region can be measured accordingly. Beam propagation analysis is carried out and confirms that significant scattering and multiple reflections occur in the channel as shown in Fig. 6. It is worth notifying that after the transverse channel, a large portion of power is still confined in the fiber core enabling power measurements.
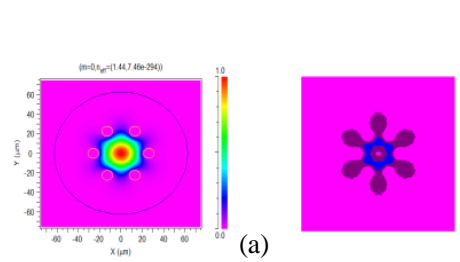

(a)

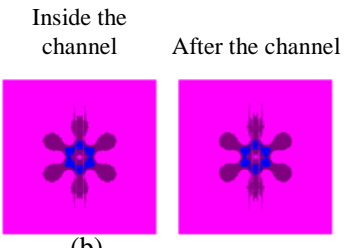

(b)

Fig. 5. (a) Fundamental mode of the holey fiber, (b) Mode evolution in holey fiber with a transverse microchannel.

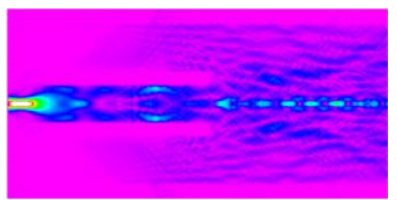

Fig. 6. Beam propagation analysis of the microfluidic holey fiber.

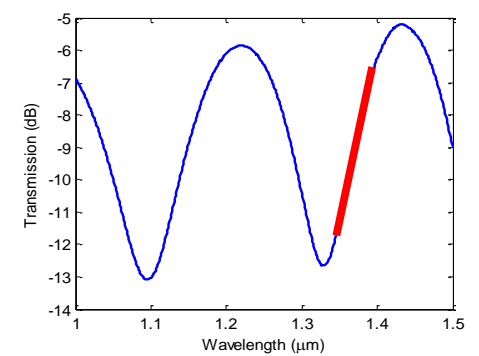

(a) Transmission as a function of refractive index of liquid in the channel

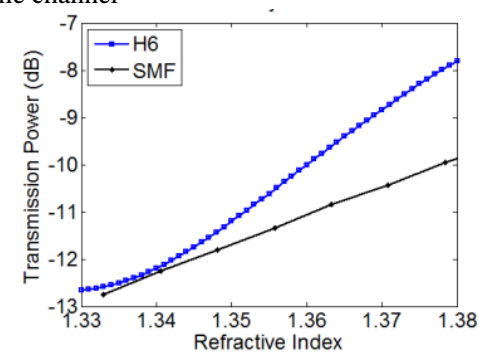

(b) Comparison between the proposed fiber and SMF in RI range $1.33-1.38$

Fig. 7. (a) The transmission power as a function of refractive index in the proposed microfluidic holey fiber structure, (b) comparison between the proposed holey fiber and SMF in the RI range of 1.33 to 1.38.

Liquid analyte and purging liquid can be easily pumped into the channel and facilitate rapid and accurate detection. The refractive index sensing can be carried out by monitoring the output power of the device. The transmitted power is varied by changing the refractive index (RI) of the liquid in the channel with a profile shown in Fig. 7(a). Please note that most fluidic sensing applications work in the range of 1.33 to 1.38 highlighted by a straight line in Fig. 7(b), the liquid RI sensitivity of the proposed device is $106 \mathrm{~dB} / \mathrm{RIU}$, i.e. the detection resolution is 9E-5 RIU if optical power detection resolution is $0.01 \mathrm{~dB}$. Figure 7(b) shows the transmitted power in the range of 1.33 to 1.38 with a comparison with SMF-based microfluidic device which has sensitivity of $56 \mathrm{~dB} / \mathrm{RIU}$ [14].

In conclusion, a holey fiber structure with a microchannel is proposed for the purpose of enhancing liquid sensing accuracy. The simulation results demonstrate that the sensitivity is twice that of the counterpart version of SMF. The proposed fibers are fabricated and also characterized. Further experiments of actual channel inscription and liquid detection measurement will be carried out to confirm the improvement of sensitivity compared to SMF microfluidic devices.

This work was supported in part by the A*STAR-SERC Thematic Strategic Research Programme (TSRP) grant 102152 0012. The authors would like to acknowledge the funding support of the A*STAR Graduate Scholarship (Post-Doctoral Fellowship). The authors would like to thank Dr Yicheng Lai from Data Storage Institute, Agency for Science, Technology and Research (A*STAR), Singapore for helpful discussion.

\section{References}

[1] J.C. Knight, J. Arriaga, T.A. Birks, A. Ortigosa-Blanch, W.J. Wadsworth, P.St.J. Russell, IEEE Photon. Technol. Lett. 12, 807 (2000).

[2] D.J.J. Hu, P. Shum, G. Ren and C. Lu, App. Opt. 47, 5061 (2008).

[3] P.St.J. Russell, P. Hölzer, W. Chang, A. Abdolvand, J.C. Travers, Nature Phot. 8, 278 (2014).

[4] D.J.J. Hu, P. Shum, C. Lu, G. Ren, Opt. Comm. 282, 4072 (2009).

[5] K. Milenko, D.J.J. Hu, P.P. Shum, T. Zhang, J. L. Lim, Y. Wang, T.R. Wolinski, H. Wei, W. Tong, Opt. Lett. 37, 1373 (2012).

[6] D.J.J. Hu, Y. Wang, J.L. Lim, T. Zhang, K. Mileńko, Z. Chen, M. Jiang, G. Wang, F. Luan, P.P. Shum, Q. Sun, H. Wei, W. Tong, T.R. Woliński, IEEE Sensors J. 12, 1239 (2012).

[7] M.T. Myaing, J.Y. Ye, T.B. Norris, Opt. Lett. 28, 1224 (2003).

[8] D.J.J. Hu, J.L. Lim, M.K. Park, L.T.-H. Kao, Y. Wang, IEEE J. Sel. Top. Quant. Electron. 18, 1293 (2012).

[9] D.J.J. Hu, J.L. Lim, Y. Cui, K. Milenko, Y. Wang, P.P. Shum, T.R. Wolinski, IEEE Photon. J. 4, 1248 (2012).

[10] Y. Cui, P.P. Shum, D.J.J. Hu, G. Wang, G. Humbert, X.-Q Dinh, IEEE Photon. J. 4, 1801 (2012).

[11] D.J.J. Hu, P.P. Shum, J.L. Lim, Y. Cui, K. Milenko, Y. Wang, T. Wolinski, IEEE Photon. J. 4, 2010 (2012).

[12] U.S. Dinish, Y.F. Chit, S.S. Kiat, B. Ramaswami, A. Kumar, M. Olivo. Biosensors and Bioelectronics 33, 293 (2012).

[13] K. Zhou, Z. Yan, L. Zhang, I. Bennion, Opt. Expr. 19, 11769 (2011).

[14] J. Petrovic, Y. Lai, I. Bennion, Appl. Opt. 47, 1410 (2008). 